\title{
Variation in the proportion of flower visitors of Arum maculatum along its distributional range in relation with community-based climatic niche analyses
}

\author{
Anahí Espíndola, Loïc Pellissier and Nadir Alvarez \\ A. Espindola (maria.espindola@unine.ch), Laboratory of Evolutionary Entomology, Inst. of Biology, Univ. of Neuchâtel, Emile-Argand 11, \\ CH-2000 Neuchâtel, Switzerland. - L. Pellissier and N. Alvarez, Dept of Ecology and Evolution, Univ. of Lausanne, Biophore Building, \\ CH-1015 Lausanne, Switzerland.
}

\begin{abstract}
Because species-specific interactions between plants and insects require considerable physiological adaptations to establish and be maintained through time and space, highly specialized interactions are rare in nature. Consequently, even if some one-to-one interactions might appear locally specialized, additional partners may be involved at a wider scale.

Here, we investigate the geographical constancy in the specificity level of the specialized lure-and-trap pollination antagonism involving the widespread European Arum maculatum and its associated Psychodid pollinators. Until now, studies concurred in demonstrating that one single insect species, Psychoda phalaenoides, efficiently cross-pollinated plants; researches were, however, performed locally in western Europe. In this study we characterize for the first time the flower visitors' composition at the scale of the distribution range of $A$. maculatum by intensively collecting plants and insects throughout the European continent. We further correlate local climatic characteristics with the community composition of visiting arthropods.

Our results show that flowers are generally visited by $P$. phalaenoides females, but not over the whole distribution range of the plant. In some regions this fly species is less frequent or even absent and another species, Psycha grisescens, becomes the prevailing visitor. This variability is geographically structured and can be explained by climatic factors: the proportion of $P$. grisescens increases with higher annual precipitations and lower precipitations in the warmest trimester, two characteristics typical of the Mediterranean zone. Climate thus seems driving the specificity of this interaction, by potentially affecting the phenology of one or both interacting species, or even of volatile and heat production in the plant. This result therefore challenges the specificity of other presumably one-to-one interactions covering wide distribution ranges, and provides an example of the direct effect that the abiotic environment can have on the fate of plant-insect interactions.
\end{abstract}

In its early definition, the coevolutionary theory predicted that adaptive responses and speciation patterns in organisms were strongly influenced by one or a few interacting species (Ehrlich and Raven 1964). This has led to a simple view of coevolution, in which the evolutionary history of interacting organisms mostly occurs in a strict framework of coadaptation and cocladogenesis, as formerly proposed in the case of figs and fig-wasps (Ramirez 1970, Jousselin et al. 2008). Establishing highly specific relationships would thus represent an evolutionary stable strategy, since it would imply a rise in the efficiency of exploitation, and would assure the constancy through time of the resource(s) exploited (Bronstein 2009). However, such cases of speciesspecific interactions between plants and insects are more the exception than the rule (Hoeksema and Bruna 2000) and the view of strict coevolution evolved towards a more complex and diffuse coevolutionary concept taking place at the community level (Stanton 2003). Reasons for the scarcity of one-to-one interactions include the fact that specific biological associations require the development of particular ecological and physiological features in at least one of the interacting lineages (Brodie and Ridenhour 2002), which might entail substantial energy investments. Moreover, specialization could also cause a loss of efficiency in the exploitation of other non-specific associated species (Thompson 1994), which potentially makes these interactions sensitive to the loss of their specific partners. Thompson's theory on the geographic mosaic of coevolution (Thompson 2005) also predicts specialized interactions to be scarce in communities characterized by varying or disturbed environments: because biological interactions are expected to change along the distributional gradient, partner variations and multiple associations (McKinney 1997, Débarre and Gandon 2010) could be favoured and specialization might be then evolutionarily non-adaptive. Even if Thompson's view is sound, investigating simultaneously environmental variations and interactions is, in practice, a complex challenge. As a consequence, the environment-interaction relationship has been 
only analyzed in a few multi-partners case-studies (reviewed by Laine 2009). In contrast, specific interactions provide simple and adequate natural laboratories for this type of studies, because of the restricted number of interacting species.

The genus Arum (Araceae) is characterized by the display of specialized antagonistic pollination interactions with arthropods, which have been documented for all but one species (Gibernau et al. 2004, Diaz and Kite 2006, Lobin et al. 2007): insects visit the plant but no service or resource is provided in exchange for pollination. One of the most studied pollination systems in the genus is that of lordsand-ladies Arum maculatum, a widely distributed European species (Boyce 2006, Espíndola et al. 2010). Its monoecious and protogynous inflorescences are formed by a spadix surrounded by a spathe, a characteristic feature in Araceae (Mayo et al. 1997). The lower part of the spathe is narrowed and encloses the fertile flowers, forming the floral chamber. During anthesis the chamber opening is covered by modified male flowers, the staminodes (Boyce 1993), which thus close the only aperture. In combination to these morphological features, plants of this species present a complex combination of heating cycles and odour production, which help lure small coprophillous Psychodid flies. Duped flies are attracted to the smelling flower and, after landing on the oily spathes, slip into the floral chamber. Because of the staminodes, they cannot fly away, being thus trapped for several hours. While trying to flee they move in the chamber and will - if they had been recently trapped by another Arum - passively pollinate the receptive female flowers. After the female phase, pollen release takes place and the trapped hairy flies are covered with it. Once pollen is liberated, staminodes wither, walls lose their oily nature and the captive insects are set free (Lack and Diaz 1991).

Among the Arum species known to show lure-and-trap pollination, A. maculatum has called the attention of ecologists for decades notably because it appears to display a highly specific pollination biology (Beck 1983, Lack and Diaz 1991, Diaz and Kite 2002). Whereas other Arum species are pollinated by a guild of visiting insects (Gibernau et al. 2004), investigations concurred in demonstrating that $A$. maculatum relied only on the fly species Psychoda phalaenoides (Diptera: Psychodidae) (Diaz and Kite 2002). Even if researchers have also identified other species (mainly Smittia pratorum (Diptera: Chironomidae) and Psycha grisescens (Diptera: Psychodidae)) trapped in floral chambers, these were present in very small proportions compared to $P$. phalaenoides, and because they were carrying significantly less pollen grains than the former it was assumed that their occurrence in the flower was accidental (Diaz and Kite 2002). Since at the several locations studied in western Europe the species composition of visiting insects was similar, and because in all sites $P$. phalaenoides was by far the most frequent visitor, it became tacitly accepted that the interaction between the plant and $P$. phalaenoides was species-specific (Gibernau et al. 2004) and constant all over the A. maculatum distribution range. However, the plant is distributed across a much wider area than that studied since it covers a large part of the occidental Palearctic from Spain to Denmark and from Ireland to Bulgaria (Boyce 2006) and thus potentially spans a wide diversity of climatic conditions and environments.
The theory of the geographic mosaic of coevolution would therefore predict that specialization should not be maintained throughout the plant's distribution range, and that the identity of pollinators might change in space, along environmental conditions. In order to test such predictions, we aim in the present work at: 1) evaluating the visitors composition of $A$. maculatum inflorescences with an unprecedented coverage of the whole plant's distribution range; 2) analyzing to what extent the putative spatial variation in visitors identities is correlated with environmental variations associated with climatic factors.

\section{Material and methods}

\section{Sampling and insect identification}

Fifty-six populations of Arum maculatum were sampled across the whole plant's distribution range (Supplementary material Appendix 1) during springs 2006, 2007 and 2008. To avoid biases related either to regional differences in the sampling effort or to local inter-annual variation, populations were sampled when possible based on a $200 \mathrm{~km}$ grid (not shown), and all main European regions (i.e. central and northern Europe, Balkans, Italian Peninsula) were visited all years. Plant populations were sampled during blooming period and depending on populations sizes, up to ten floral chambers per population were randomly selected and collected (Supplementary material Appendix 1). Floral chambers and their visitors were independently kept in 70\% ethanol. Insects were identified when possible to species with the help of entomological keys (Chinery 1976, Withers 1988, Ježek 1990, Oosterbroek 2006). Arthropods were counted, and proportions were calculated per population, per floral chamber and for the totality of the samples.

\section{Spatial and environmental structure in the composition of visitors}

Spatial variation of the floral chamber fauna was analyzed using ArcMap 9.3 (ESRI, Redlands CA, USA) by plotting and examining the compositions of all sampled visitors. To test for climatic factors influencing the composition of visitors found in the floral chambers, we performed a canonical correspondence analysis (CCA) (ter Braak 1986) using the library ade4 (Thioulouse et al. 1997) on R 2.10.0 (R Development Core Team 2010). The CCA is designed to extract synthetic environmental gradients from ecological data sets, by describing the differential habitat preferences (i.e. from a climatic point of view in our study) of taxa via an ordination diagram. Climatic values were extracted for each sampled point from seven WORLDCLIM layers at a resolution of 2.5 arc-minutes (Hijmans et al. 2005). Layers were chosen based on their ability to describe basic general characteristics of the environment (mean annual and seasonal temperatures and precipitations). Finally, the CCA was calculated on visitor abundances at different taxonomic levels (classes, orders, families, different species of Psychodids; Supplementary material Appendix 1).

Simultaneously, in order to identify the climatic factors explaining the variation in abundances of the two most 
frequent and constant visiting species (i.e. Psychoda phalaenoides and Psycha grisescens), we calculated their proportion at each population, and adjusted a general linear model (GLM) with a binomial distribution considering the climatic variables previously extracted. Finally, the significance of the parameters estimated was tested by a Wald-z statistic, as implemented in the function glm in R 2.10.0.

\section{Results}

\section{General composition of visitors}

Two hundred and eighty-four floral chambers were collected from the 56 visited locations, containing a total of 11036 arthropods. The mean number of arthropods trapped per population and per floral chamber were 187.05 (SE $=$ 53.22) and $38.58(\mathrm{SE}=8.03)$, respectively.

Absolute overall proportions were as follows: arthropods belonged to classes Arachnida (5.53\%) and Insecta (94.47\%). The totality of Arachnida was represented by the subclass Acarina. Insecta were composed of orders Diptera (92.68\%), Collembola (1.21\%), Hemiptera $(0.38 \%)$ and Coleoptera $(0.19 \%)$. The far most important proportion of Diptera was formed by the family Psychodidae (87.49\%) in which main visitors were females of Psychoda phalaenoides (84.27\%), followed by Psycha grisescens from both genders $(2.06 \%$ females and $0.70 \%$ males); these two species together represented $87.03 \%$ of all arthropods trapped in the flower chambers. The remaining Psychodid species were very little represented and accounted for $0.46 \%$ of the total sampling.

Based on these results and in order to identify general patterns in the composition of visiting insects, sampled locations were classified according to the relative numbers of the two most abundant species P. phalaenoides and P. grisescens: locations comprising more $P$. phalaenoides than $P$. grisescens were defined as 'p' sites, while locations comprising more P. grisescens than P. phalaenoides were considered as 'g' sites (Supplementary material Appendix 2). As shown in Fig. 1, sites ' $\mathrm{p}$ ' and ' $\mathrm{g}$ ' not only differed in the relative abundance of the two most frequent species but also in the composition and overall abundance of visitors. In 'p' sites, the composition was strongly dominated by females of $P$. phalaenoides, with very small proportions of other groups, and a particularly high number of visitors $(>70)$ in about half of them. In ' $\mathrm{g}$ ' sites, the number of visitors was low (a maximum of 77), both males and females of $P$. grisescens were dominant, but other insect groups were also found in non-negligible proportions (Supplementary material Appendix 1).

\section{Geographic variation in the composition of visitors}

Figure 2 shows the variation in the composition of floral chamber visitors all over the plant distribution. Psychoda phalaenoides is almost found at every location (in blue on Fig. 2) but in varying proportions. Populations in the southern edge of the sampled area include a more diverse fauna, mainly represented by $P$. grisescens (red and pink portions in Fig. 2a). Moreover, when pies are resized proportionally to the total number of visitors (Fig. 2b), a pattern appears, with populations from the south being also less visited than those from the north, and this, irrespective of the year of collection (data not shown, but see Supplementary material Appendix 1).

\section{Influence of climate on visitors' composition}

The composition of the floral chambers' fauna seems to be mainly determined by the first CCA axis $(64.95 \%$ variance explained), with P. phalaenoides, Acarina and P. setigera showing an opposite ordination to that of the other groups (Fig. 3a). It is worth noting that the correlation between the abundances of the two most commonly found species (i.e. P. phalaenoides and P. grisescens) is strongly negative. Regarding climatic variables (Fig. 3b), 'p'-type and 'g'-type populations are differentiated by temperatures, which are negatively correlated to the amount of precipitations: 'g'-type populations are associated with higher temperatures and lower rain rates than are 'p'-type.

The GLM shows that proportions of the two main visitors $P$. phalaenoides and $P$. grisescens are explained by annual precipitations $(\mathrm{p}=0.023$; Table 1$)$ and precipitations during the warmer quarter $(\mathrm{p}=0.007$; Table 1$)$. The proportion of $P$. grisescens increases with higher annual precipitations and lower precipitations during the warmer quarter. The opposite effect is observed for $P$. phalaenoides.

\section{Discussion}

\section{A more complete insight on the composition of A. maculatum pollinators}

Species-specific interactions are rare, because their persistence strongly relies on tight and durable coevolutionary forces. Such conditions are particularly difficult to maintain

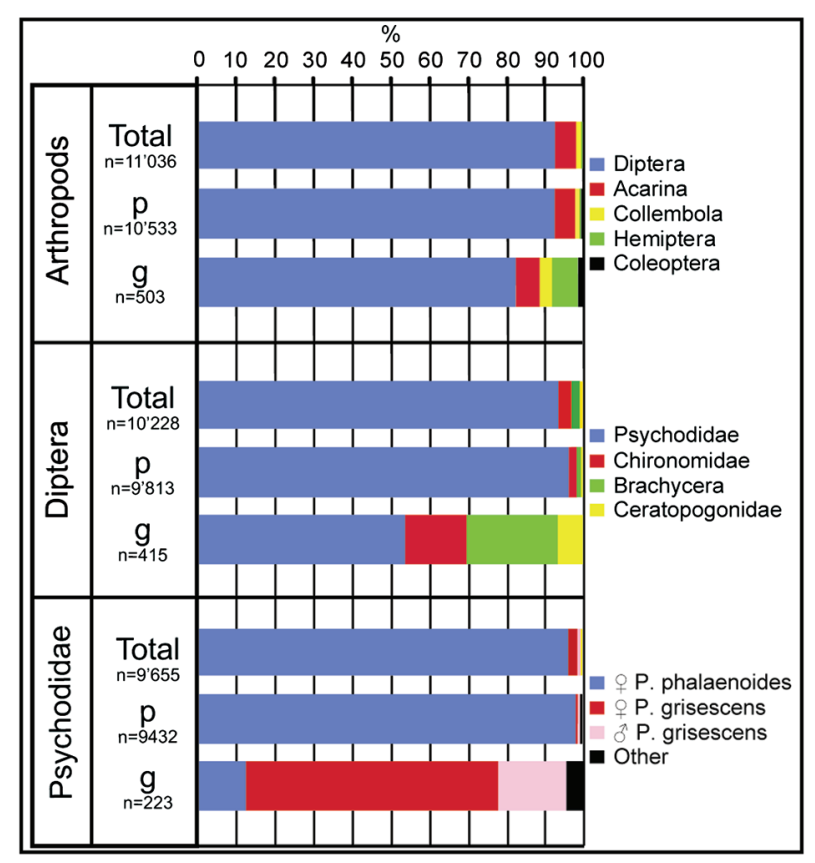

Figure 1. Proportions of Arthropods, Diptera and Psychodidae found in floral chambers. Total numbers according to the two pollinators' community categories are indicated (i.e. 'p': populations harbouring more $P$. phalaenoides than $P$. grisescens; ' $\mathrm{g}$ ': populations harbouring more $P$. grisescens than $P$. phalaenoides; see text). Legend is shown at the right of the corresponding bars. 

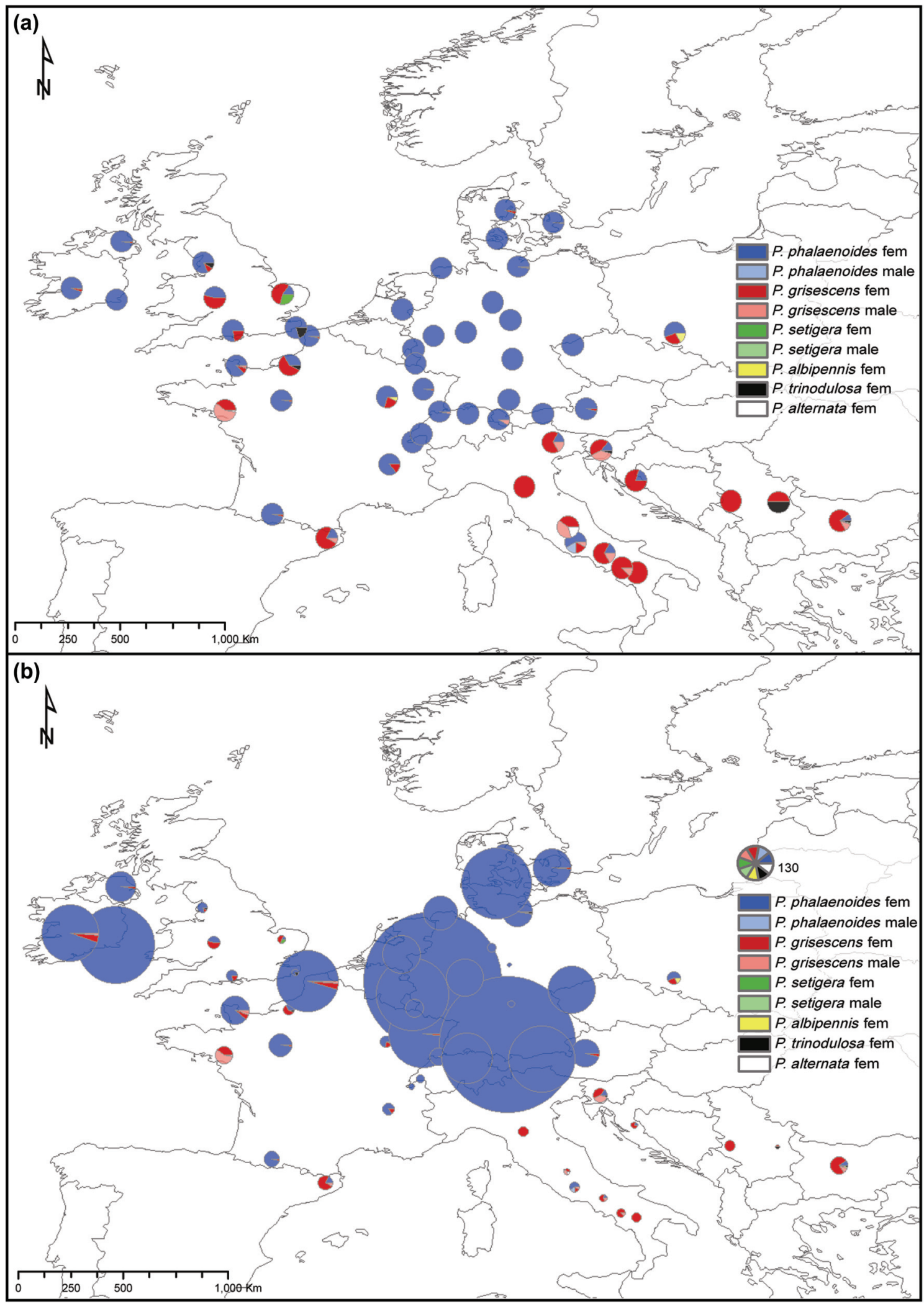

Figure 2. Distribution and composition of Psychodids found in floral chambers. Colours indicate species and sex. (a) proportions of Psychodids per population. (b) proportions resized in function of total number of trapped Psychodids (for a size reference, refer to map legend).

in species with wide distributions, in which environmental pressures are highly heterogeneous across their distribution ranges (Thompson 1994). Accordingly, our results indicate that the reported consistency in the strong and specific interaction between $A$. maculatum and its main pollinator is not preserved throughout the whole distribution range of the plant: both the total number of visitors and their composition are structured spatially (Fig. 2). Overall, there was 


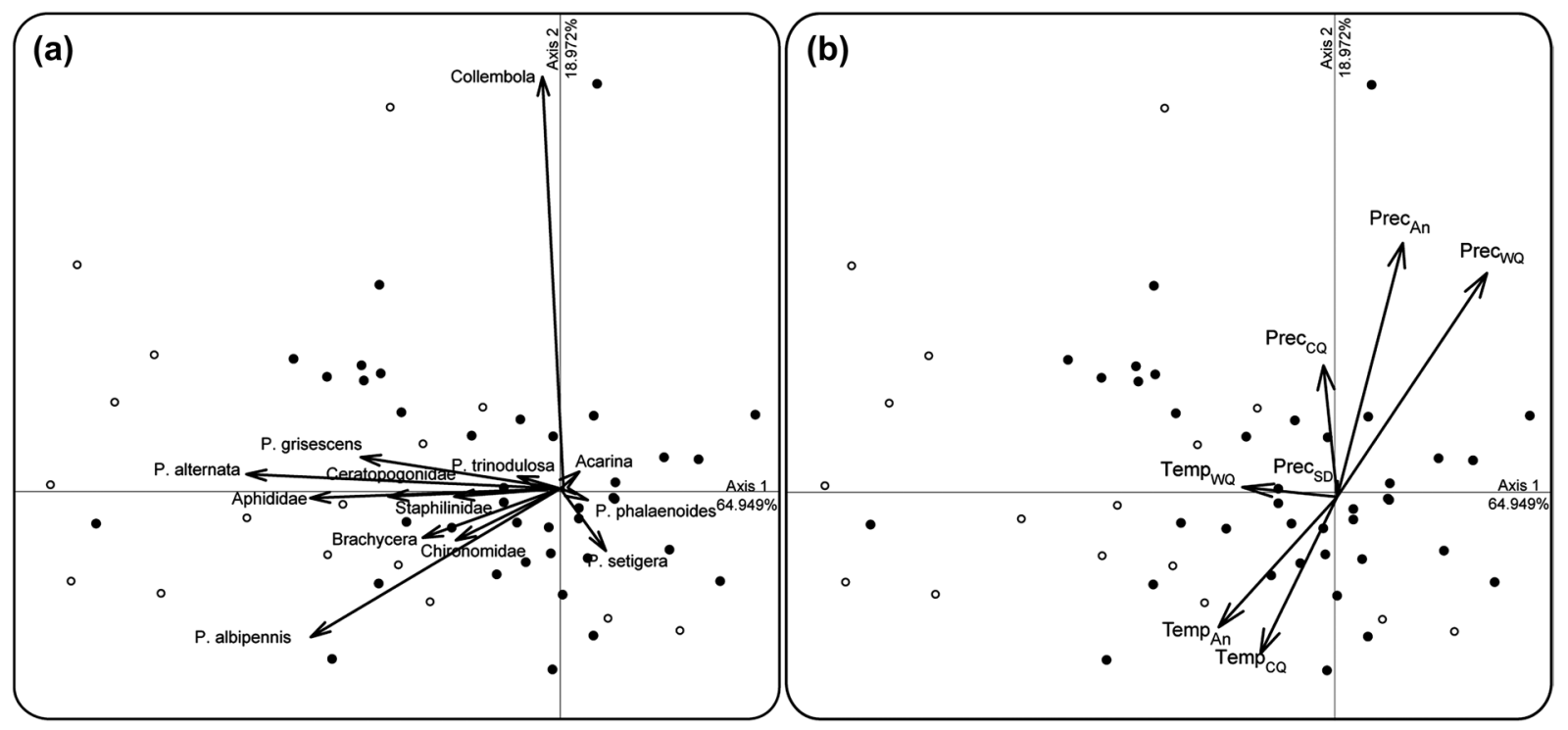

Figure 3. Canonical correspondence analysis for (a) the number and composition of arthropods found in floral chambers and (b) climatic variables. Variables are indicated on each arrow; direction of arrows indicates the sense of correlations. Filled circles represent type-' $\mathrm{p}$ ' populations, while open circles refer to type-'g' populations. Percentage of variance explained is shown on axes. Refer to Table 1 for explanations on variables in (b).

a large variation in the abundance of $P$. phalaenoides specimens at each sampled site (ranging from zero to more than a thousand, Fig. 2b). Populations harbouring high numbers of $P$. phalaenoides females were mainly found in central and northern Europe, whereas those hosting a lower number of visitors - generally more diverse and mainly composed of P. grisescens males and females $(82.96 \%)$ - were found in the Mediterranean area as well as in northern France (Fig. 2, Supplementary material Appendix 2). The similar behaviours, morphologies and ecologies shared by $P$. phalaenoides and $P$. grisescens probably attest to the ability of the latter in transporting A. maculatum pollen. However, the pollination effectiveness of $P$. grisescens should be investigated in these populations before unambiguously confirming its identity as a real pollinator. Because $A$. maculatum can also reproduce by clonal means (Boyce 1993), further population genetics analyses in the plant all over its southern range of distribution could provide information on its level of crosspollination and consequently, on the ability of $P$. grisescens to efficiently transfer pollen from one plant to another. Finally, other arthropods captured by $A$. maculatum were less abundant and were not structured geographically (Fig. 2). They appear to be more casual and accidental visitors than

Table 1. Estimates, z-values and associated p-values of the GLM adjusted to the proportion of $P$. grisescens and $P$. phalaenoides. $*: p<0.05$.

\begin{tabular}{|c|c|c|c|}
\hline Climatic variable & Estimate & z-value & $\mathrm{p}$-value \\
\hline Intercept & -4.793 & -0.74 & 0.460 \\
\hline Mean annual temperature $\left(\operatorname{Temp}_{\mathrm{Ann}}\right)$ & 0.131 & 1.026 & 0.305 \\
\hline Annual precipitations $\left(\operatorname{Prec}_{\mathrm{Ann}}\right)$ & 0.024 & 2.27 & $0.023 *$ \\
\hline Temp. warmest quarter $\left(T p_{w Q}\right)$ & -0.040 & -0.514 & 0.607 \\
\hline Temp. coldest quarter $\left(\mathrm{Temp}_{\mathrm{CQ}}\right)$ & -0.084 & -1.487 & 0.137 \\
\hline Precip. SD $\left(\operatorname{Prec}_{S D}\right)$ & 0.062 & 1.155 & 0.248 \\
\hline Precip. warmest quarter $\left(\operatorname{Prec}_{\mathrm{WQ}}\right)$ & -0.052 & -2.79 & $0.005^{*}$ \\
\hline Precip. coldest quarter $\left(\operatorname{Prec}_{\mathrm{CQ}}\right)$ & -0.048 & -1.801 & 0.072 \\
\hline
\end{tabular}

reliable pollen vectors, but this does not exclude their potential importance in cross-pollination when main visitors lack.

\section{Variation in the attracted insect genders}

Our study points out that $A$. maculatum flowers capture mainly females of $P$. phalaenoides, but both sexes of P. grisescens (Fig. 1). This result is in agreement with the findings of Diaz and Kite (2002), who described a similar trend in southern England. It is therefore likely that the odour cues released by the plant are different or are interpreted differently by the two species. Whereas $P$. phalaenoides females are attracted by $A$. maculatum odours that mimic their laying substrate (i.e. cow-dung; Kite 1995), one could imagine that this same odour also represents a mating site for $P$. grisescens (at least in males). This would be plausible since cases in which insects mate at their laying sites are not rare (Crowson 1981, Petit et al. 2007).

\section{The effect of the environment on plant-insect interactions}

Our results show that the composition of arthropods found in floral chambers of $A$. maculatum is correlated with climatic variables (Fig. 3, Table 1). The two most abundant visitors (i.e. $P$. phalaenoides and $P$. grisescens) were negatively correlated in the CCA, indicating that there is a strong differentiation of populations visited by one or another species in response to environmental conditions (Fig. 3a): populations dominated by $P$. grisescens (type ' $\mathrm{g}$ ', open circles in Fig. 3) were mainly clustered in regions displaying higher summer temperatures combined with lower rain rates. This pattern is also confirmed by the GLM results, in which annual precipitations and precipitations during the warmest quarter were significantly correlated (positively and negatively, respectively) with the relative proportions of $P$. grisescens and P. phalaenoides. 
It appears that precipitation-related factors modify the visitors' composition and do therefore affect the dynamics of the interaction between $A$. maculatum and its pollinators. These results tend to show that predictions from the theory of the geographic mosaic of coevolution (i.e. variation of the interaction in a variable environment) apply to the case of $A$. maculatum. This is, to our knowledge, one of the few studies (see also Toju 2008) in which the expectations of Thompson's theory match the observed pattern in the context of interacting species, and the first time it is demonstrated in plant-insect antagonistic interactions beneficial to the plant.

The proximal factors underlying this effect might be explained by three non-mutually exclusive hypotheses. First, because most ' $\mathrm{g}$ ' sites were found in the Mediterranean region - which is characterized by rain regimes different from those of temperate Europe, with at least one marked dry season (Quézel 1985) - water stress might influence the plant phenology, leading it to flower at different time periods, uncoupled with the life cycle of $P$. phalaeonoides. As a consequence, it is possible that when Mediterranean A. maculatum blossom, their main pollinator $P$. phalaenoides is not present yet/anymore. Second, differences in precipitations could have an impact on the physiology of volatile and heat production, which are known to be both energetically costly (Minorsky 2003) and a key-factor for the attraction of pollinators in this system. Since changes in rain regimes and rates can put plants under water stress and modify their metabolism (Gouinguene and Turlings 2002, Baldwin et al. 2006), one could imagine that $A$. maculatum plants from sites visited mainly by $P$. grisescens present different odour/ heat patterns than those visited mainly by $P$. phalaenoides, the latter relying on a much more constant water supply. These differences could make 'g'-type populations less successful than 'p'-type populations in terms of attraction of the most efficient pollinator (i.e. P. phalaenoides), this would also be in agreement with both their lower number of visitors and higher arthropod species diversity. Finally, it is also possible that changes in water resources modify the insects' life cycles, limiting their phenological overlap with plants blossom. This seems however unlikely, since faunistic records indicate that both fly species are always present long before and after the flowering period, and over a larger distribution range than that of the plant (Ježek and Barták 2000, Fauna Europaea 2004). An alternative would be that even if $P$. phalaenoides is known to occur in the Mediterranean region, this area constitutes a marginal habitat of lower suitability, in which the species is rather rare compared to more northern habitats. In order to test this hypothesis, further intensive entomological collections should be performed all over the insect's distribution range.

\section{Conclusion}

In this study we show that arthropod visitors of $A$. maculatum are mainly restricted to the two Psychodids $P$. phalaenoides and $P$. grisescens. Overall, as previously observed at a more limited geographical scale (Beck 1983, Diaz and Kite 2002), P. phalaenoides females are the most abundant visitors of $A$. maculatum flowers. However, the abundance and composition of the fauna captured in floral chambers strongly varies across the distribution range of the plant, with $P$. grisescens becoming notably more abundant in the Mediterranean region. As a consequence, diversity and abundance of arthropods are geographically structured (without any notable inter-annual effect), indicating that all over its distribution range the plant might exploit at least two species and not a single one, as previously assumed. Our results show that variation in environmental conditions, particularly in rain regimes, is correlated with changes in the visitors' composition. This climatic effect could be explained by the role played by water stress in modifying the phenology of either the plant or the insect, or the physiology of volatile and heat production in the plant. Precipitation-related factors therefore affect not only the visitors' composition of the plant, but also the specificity of the pollination interaction.

This study provides evidences confirming predictions of the theory of the geographic mosaic of coevolution, directly demonstrating that environmental variation does have an impact on antagonistic interactions. It finally points out the importance of applying large-scale surveys when challenging the existence of putative one-to-one interactions.

Acknowledgements - The authors would like to thank Dr. Z. Kącki and Dr. Z. Dajdok (Univ. of Wrocław, Poland), Dr. M. Gibernau (UMR CNRS, Univ. Paul Sabatier; France), Dr. J. Ježek (Natl Mus. of Prague; Czech Republic), Dr. F. Conti (Centro Ricerche Floristiche dell'Appennino, Italy), Dr. A. Diaz (Bournemouth Univ., England), N. Revel, Dr. Y. Triponez and P. Zryd (Univ. of Neuchâtel, Switzerland) for their precious assistance during sampling and data analysis. We are grateful to the journal editor A. Traveset, M. Méndez and A. Diaz for constructive comments on a previous version of this manuscript. This study was funded by the Swiss National Science Foundation (project no. 3100A0-116778).

\section{References}

Baldwin, I. T. et al. 2006. Volatile signaling in plant-plant interactions: "Talking trees" in the genomics era. - Science 311: 812-815.

Beck, I. 1983. Blütenökologie Untersuchungen am Aronstab (Arum maculatum L.). Spezielle Botanik. - Univ. of Tübingen, p. 123.

Boyce, P. C. 1993. The genus Arum. - R. Bot. Gard. Kew.

Boyce, P. C. 2006. Arum - a decade of change. - Aroideana 29: $132-137$.

Brodie, E. D. and Ridenhour, B. J. 2002. Reciprocal selection at the phenotypic interface of coevolution. - Integr. Compar. Biol. 42: 1202-1202.

Bronstein, J. L. 2009. The evolution of facilitation and mutualism. - J. Ecol. 97: 1160-1170.

Chinery, M. 1976. Les insectes d'Europe. - Elsevier Séquoia.

Crowson, R. A. 1981. The biology of the Coleoptera. - Academic Press.

Débarre, F. and Gandon, S. 2010. Evolution of specialization in a spatially continuous environment. - J. Evol. Biol. 23: 1090-1099.

Diaz, A. and Kite, G. C. 2002. A comparison of the pollination ecology of Arum maculatum and A. italicum in England. - Watsonia 24: 171-181.

Diaz, A. and Kite, G. C. 2006. Why be a rewarding trap? The evolution of floral rewards in Arum (Araceae), a genus characterized by saprophilous pollination systems. - Biol. J. Linn. Soc. 88: 257-268.

Ehrlich, P. R. and Raven, P. H. 1964. Butterflies and plants: a study in coevolution. - Evolution 18: 586-607. 
Espíndola, A. et al. 2010. New insights into the phylogeny and biogeography of Arum L. (Araceae): unravelling its evolutionary history. - Bot. J. Linn. Soc. 163: 14-32.

Fauna Europaea. 2004. Fauna Europaea ver. 1.1. <www.faunaeur. org $>$. Accessed 10 January 2010.

Gibernau, M. et al. 2004. Pollination in the genus Arum - a review. - Aroideana 27: 148-166.

Gouinguene, S. P. and Turlings, T. C. J. 2002. The effects of abiotic factors on induced volatile emissions in corn plants. - Plant Physiol. 129: 1296-1307.

Hijmans, R. J. et al. 2005. Very high resolution interpolated climate surfaces for global land areas. - Int. J. Climatol. 25: 1965-1978.

Hoeksema, J. D. and Bruna, E. M. 2000. Pursuing the big questions about interspecific mutualism: a review of theoretical approaches. - Oecologia 125: 321-330.

Ježek, J. 1990. Redescriptions of nine common palaearctic and holarctic species of Psychodini End. (Diptera: Psychodidae). - Acta Entomol. Mus. Natl Pragae 43: 33-83.

Ježek, J. and Barták, M. 2000. Psychodidae. - Folia Fac. Sci. Nat. Univ. Masaryk. Brun. 104: 93-100.

Jousselin, E. et al. 2008. One fig to bind them all: host conservatism in a fig wasp community unraveled by cospeciation analyses among pollinating and nonpollinating fig wasps. - Evolution 62: 1777-1797.

Kite, G. C. 1995. The floral odour of Arum maculatum. - Biochem. Syst. Ecol. 23: 343-354.

Lack, A. J. and Diaz, A. 1991. The pollination of Arum maculatum L. - a historical review and new observations. - Watsonia 18: 333-342.

Laine, A. L. 2009. Role of coevolution in generating biological diversity: spatially divergent selection trajectories. - J. Exp. Bot. 60: 2957-2970.

Supplementary material (available online as Appendix O18937 at $<$ www.oikos.ekol.lu.se/appendix $>$ ). Appendix 1-2.
Lobin, W. et al. 2007. A new Arum species (Areae, Araceae) from NE Turkey and Georgia. - Willdenowia 37: 445-449.

Mayo, S. J. et al. 1997. The genera of Araceae. - R. Bot. Gard. Kew.

McKinney, M. L. 1997. Extinction vulnerability and selectivity: combining ecological and paleontological views. - Annu. Rev. Ecol. Syst. 28: 22.

Minorsky, P. V. 2003. The hot and the classic. - Plant Physiol. 132: 25-26.

Oosterbroek, P. 2006. The European families of the Diptera. Identification, diagnosis, biology. - KNNV Publishing.

Petit, D. et al. 2007. On the mating and laying sites of Uromenus brevicollis ssp. insularis in Corsica (Ensifera, Tettigoniidae). - J. Orthoptera Res. 16: 145-151.

Quézel, P. 1985. Definition of the Mediterranean region and the origin of its flora. - Trends Ecol. Evol. 23: $564-571$.

Ramirez, W. B. 1970. Host specificity of fig wasps (Agaonidae). - Evolution 24: 680-690.

Stanton, M. L. 2003. Interacting guilds: moving beyond the pairwise perspective on mutualisms. - Am. Nat. 162: S10-S23.

ter Braak, C. J. F. 1986. Canonical correspondence analysis: a new eigenvector technique for multivariate direct gradient analysis. - Ecology 67: 1167-1179.

Thioulouse, J. et al. 1997. ADE-4: a multivariate analysis and graphical display software. - Stat. Comput. 7: 75-83.

Thompson, J. N. 1994. The coevolutionary process. - Univ. of Chicago Press.

Thompson, J. N. 2005. Coevolution: the geographic mosaic of coevolutionary arms races. - Curr. Biol. 15: 992-994.

Withers, P. 1988. Moth flies. Diptera: Psychodidae. - Dipterist Digest 4: 83 . 